\title{
On The Molecular Mechanism of the Calcium-Induced Gelation of Pectate. Different Steps in the Binding of Calcium Ions by Pectate. ${ }^{\dagger}$
}

\author{
Ivan Donati ${ }^{1, *}$, Julio C. Benegas ${ }^{2}$, and Sergio Paoletti ${ }^{1, \S}$ \\ ${ }^{1}$ Department of Life Sciences, University of Trieste, Via Giorgieri 5, I 34127 Trieste, Italy. ${ }^{2}$ Department \\ of Physics-IMASL, National University of San Luis, 5700 San Luis, Argentina
}

\section{Supporting Information}

\section{Section 1. Experimental details}

a) equilibrium dialysis at different $\mathrm{Ca}^{2+}$ ions concentration ${ }^{1}$

The equilibrium dialysis experiments were carried out using cellulose tubing (Kalle AG, Wiesbaden), MWCO 10'000. Prior to use, these membranes were treated with acetic anhydride, repeatedly washed with hot dilute solutions of EDTA and $\mathrm{NaHCO}_{3}$, respectively, then with ethanol, and finally rinsed thoroughly with twice distilled water. Experiments were performed at different values of $\mathrm{R}_{\mathrm{j}}$ for each value of the polymer concentration, $C p$. A treated tubing was closed by knots at the two ends. The second knot was sealed after filling the tubing with a known volume of the solution containing polymer and calcium (at $\mathrm{R}_{\mathrm{j}}$ ) (“inner" dialysis compartment). The tubing was hanged inside a large glass cylinder containing a known volume of solution containing a known concentration of calcium, whose value was estimated to be close to the final, equilibrium value of the "free" calcium ions at the given value of $\mathrm{R}_{\mathrm{j}}$ ("outer" dialysis compartment). The glass cylinder containing the two compartments was placed on a magnetic stirrer to ensure homogeneity for at least an overnight period. (Control experiments had proved that equilibrium was achieved after four hours). For checking, the volumes of the solutions of the "inner" and "outer" compartments were measured after equilibrium. In any case, the variation from the initial values was usually negligible. For each equilibrium dialysis experiment, the concentration of calcium in the outer dialysis compartment (the concentration of "free" divalent counterions, corresponding to $\left(1-r^{o s m}\right) \cdot C p$ according to equation $<1>$ ) was 
determined by complexometric titration using ethylenediaminetetraacetic acid (EDTA) as the complexing agent and Eriochrome Black T (ErioT) as the indicator.

b) membrane osmometry at different $\mathrm{Ca}^{2+}$ ions concentration ${ }^{1}$

The osmotic pressure experiments were carried out using the high-speed microvolume membrane osmometer model CSM-2 by Melabs, Inc., Palo Alto, California, 94304 USA (now Wescan Instruments, Santa Clara, CA), using internal temperature control to $\pm 0.02{ }^{\circ} \mathrm{C}$. The membranes were cut from the cellulose tubing treated as described hereabove in a). Equilibrium dialysis experiments were carried as above described out at different values of $R_{j}$ (and then $\left.r^{o s m}\right)$, using in the "inner" compartment a polymer concentration value in the range $2.5-3.0$ $\mathrm{g} \cdot \mathrm{L}^{-1}$. After equilibration, usually three new polymer-containing solutions were obtained from the starting one using the "outer" solution as the diluting solvent, to ensure chemical equilibrium of the diffusible species. At the end, altogether four solutions were prepared to be measured in the osmometer. Eventually, plots of the osmotic pressure data as a function of $C p$ were obtained at each value of $r^{o s m}$ and analyzed according to the standard equation:

$$
\pi /\left(R \cdot T \cdot C_{p}\right)=\bar{M}_{n}^{-1}+A_{2} \cdot C_{p}
$$

In the case of the experiments carried out as a function of $r^{o s m}$, the values of both $\bar{M}_{n}$ and $A_{2}$ depended of $r^{\text {osm }}$ (see Figure 1 a). In all cases, excellent linearity was observed.

c) determination of the number-average relative molar mass of $\mathrm{Na}^{+}$-pectate ${ }^{1}$

The osmotic pressure experiments on aqueous solutions of sodium pectate at two $\mathrm{pH}$ values were carried out according to the procedures described hereabove. The reduced osmotic pressure data of reference ${ }^{1}$ have been re-plotted in Figure S1. They pertain to sodium pectate in aqueous $0.1 \mathrm{M} \mathrm{NaClO}_{4}$ at $300.15 \mathrm{~K}$, measured after dialysis equilibration at two values of $\mathrm{pH}: 3.5$ and 6.5. Those two values correspond to the ordered and to the disordered conformation of pectate, respectively, as discussed in reference. ${ }^{2}$ 


\section{Figure S1}

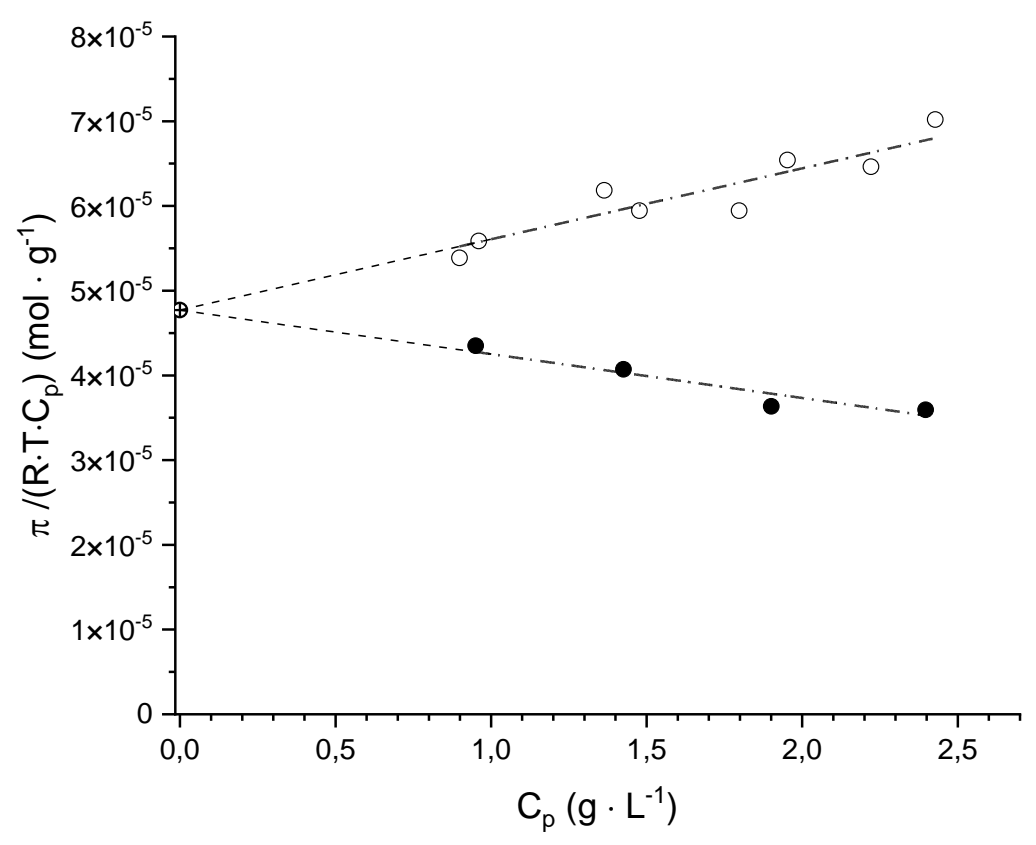

Figure S1: Dependence of the reduced osmotic pressure on the polymer concentration, $C_{\mathrm{p}}$, of sodium pectate in $0.1 \mathrm{M}$ ionic strength at $300.15 \mathrm{~K}$. Open circles: $\mathrm{pH}$ 6.5; full circles: $\mathrm{pH} 3.5$. $\left(\bar{M}_{n}=2.1 \cdot 10^{4} \mathrm{~g} \mathrm{~mol}^{-1}\right)$. Data replotted from Reference 1.

The separate linear fitting of the data of Figure S1 according to equation $\langle\mathrm{S} 1\rangle$ has given the results reported in the following Table S1:

Table S1

\begin{tabular}{|c|c|c|c|c|c|c|c|c|c|c|c|}
\hline & \multicolumn{9}{|c|}{$\mathrm{pH} 3.5$} & \multicolumn{5}{c|}{$\mathrm{pH} 6.5$} \\
\hline $\begin{array}{c}\text { Separate } \\
\text { fitting }\end{array}$ & intercept & std.error & slope & std.error & $R^{2}$ & intercept & std.error & slope & std.error & $R^{2}$ \\
\hline & $4.85 \mathrm{E}-05$ & $2.06 \mathrm{E}-06$ & $-5.61 \mathrm{E}-06$ & $1.17 \mathrm{E}-06$ & 0.91957 & $4.69 \mathrm{E}-05$ & $2.58 \mathrm{E}-06$ & $8.80 \mathrm{E}-06$ & $1.50 \mathrm{E}-06$ & 0.85177 \\
\hline $\begin{array}{c}\text { Common } \\
\begin{array}{c}\text { intercept } \\
\text { fitting }\end{array}\end{array}$ & intercept & std.error & slope & std.error & $R^{2}$ & intercept & std.error & slope & std.error & $R^{2}$ \\
\cline { 2 - 12 } & $4.77 \mathrm{E}-05$ & n.a. & $-5.18 \mathrm{E}-06$ & $3.05 \mathrm{E}-07$ & 0.99944 & $4.77 \mathrm{E}-05$ & n.a. & $8.37 \mathrm{E}-06$ & $4.26 \mathrm{E}-07$ & 0.99901 \\
\hline
\end{tabular}

Table S1: Results of the linear fitting of the reduced osmotic pressure data of sodium pectate in $0.1 \mathrm{M}$ ionic strength $\left(\mathrm{NaClO}_{4}\right)$ at $300.15 \mathrm{~K}$, at $\mathrm{pH} 6.5$ and at $\mathrm{pH}$ 3.5. Data replotted form Reference 1 (see Figure S1).

The separate fitting returns the following values for $\bar{M}_{n}: 20.6_{25} \pm 0.875$ and $21.3_{07} \pm 1.1_{69} \mathrm{~kg}$. $\mathrm{mol}^{-1}$ for $\mathrm{pH}=3.5$ and 6.5 , respectively. Given the errors, it safe to take the average value of $21.0 \pm 0.7 \mathrm{~kg} \cdot \mathrm{mol}^{-1}$ as the molar mass of sodium pectate used previously. ${ }^{1-3}$

The corresponding values of the second virial coefficient, $A_{2}$, obtained from fitting with common intercept, are: $\left(-5.1_{8} \pm 0.3_{1}\right) \cdot 10^{-3}$ and $\left(8.3_{7} \pm 0.4_{3}\right) \cdot 10^{-3} \mathrm{~mol} \cdot \mathrm{mL} \cdot \mathrm{g}^{-2}$, respectively. 
Whereas the value of $\mathrm{A}_{2}$ of the polymer at $\mathrm{pH}=6.5$ is fully in line with reported values of semiflexible (bio)polyelectrolytes with similar values of charge density, the negative value of pectate at $\mathrm{pH}=3.5$ is not surprising, given both the considerably lower charge density and the adoption of an ordered conformation (a "tight- 3 -helix"), more rigid and elongated than that neutral pH (a "loose-21-helix", as discussed in Conclusions Section of the main paper), in line with the known behavior of, e.g., polysaccharides adopting an ordered conformation. ${ }^{4}$ Moreover, the negative value of $\mathrm{A}_{2}$ at $\mathrm{pH}=3.5$ in the $\left(\mathrm{g} \cdot \mathrm{L}^{-1}\right)$ concentration range is certainly a predictor of chain association (up to gelation) which pectic acid undergoes at very low $\mathrm{pH}$ (e.g. below 2.7) and at higher concentration (e.g. $\left.30 \mathrm{~g} \cdot \mathrm{L}^{-1}\right){ }^{5}$

\section{d) intrinsic viscosity determination ${ }^{6}$}

Capillary viscosity measurements as a function of both polymer concentration and $\mathrm{Ca}^{2+}$ ion concentration were performed in the Newtonian region using an AVS 370 Schott-Geräte automatic apparatus, mounting an Ubbelohde type viscometer in a thermostat bath at $25^{\circ} \mathrm{C}$ (Schott-Geräte). The procedure for the preparation of the solutions (equilibrium dialysis, dilution, determination of the concentration of free - and bound - calcium ions, etc.) was the same as described hereabove under point $b$ ). The values of [ $\eta]$ were obtained by combined extrapolation to the common intercept $([\eta])$ of the experimental $\eta_{s p} /_{C} v s$. $C$ data and of the $\ln \left(\eta_{\text {rel }}\right) /_{C}$ vs. $C$ data, according to the Huggins:

$$
\eta_{s p} /_{C}=[\eta]+k^{\prime} \cdot[\eta]^{2} \cdot C
$$

and to the Kraemer

$$
\eta_{\text {inh }}=\ln \left(\eta_{r e l}\right) /_{C}=[\eta]+k^{\prime \prime} \cdot[\eta]^{2} \cdot C
$$

equations, respectively. $\eta_{r e l}$ is the relative viscosity, $\eta_{i n h}$ is the inherent viscosity and $\eta_{s p}\left(\eta_{s p}\right.$ $\left.=\eta_{\text {rel }}-1\right)$ is the specific viscosity. Excellent linearity was found in all cases.

e) microcalorimetric experiments

Isothermal Batch Calorimetry (IBC). Calorimetric measurements were carried out with an LKB-10700 (LKB, Bromma, Sweden) batch type IBC microcalorimeter (twin gold cells) at 25 $\pm 0.01^{\circ} \mathrm{C}$. The calorimeter is equipped with a cylinder which can rotate around the cylinder axis. Inside the cylinder two reaction vessels are placed: their signals are recorded in opposition, so that the signal from the reference is subtracted from the sample one. A schematic 
representation is given in Scheme S1. The effectiveness of this subtraction was verified in a series of parallel experiments. The signal from the calorimeter was amplified with a 182 Sensitive Digital Voltmeter from Keithley (Cleveland, OH, USA). In a typical experiment of determination of the heat of mixing, known volumes of pectate in $0.05 \mathrm{M} \mathrm{NaClO}_{4}$ and of $\mathrm{Ca}(\mathrm{ClO} 4)_{2}$ at a concentration corresponding to the desired value of $\mathrm{R}_{\mathrm{j}}$, also in $0.05 \mathrm{M} \mathrm{NaClO}_{4}$, respectively, were placed using syringes in the two compartments of the sample cell. In parallel, in the reference vessel the same volume of the $\mathrm{Ca}(\mathrm{ClO} 4)_{2}$ at a concentration corresponding to the desired value of $\mathrm{R}_{\mathrm{j}}$ in in $0.05 \mathrm{M} \mathrm{NaClO}_{4}$ was placed together with a volume of a $0.05 \mathrm{M}$ $\mathrm{NaClO}_{4}$ solution equal to the volume of the polymer-containing solution in the sample cell. In this way, the experimentally recorded signal of each heat of mixing experiment at any given value of $\mathrm{R}_{\mathrm{j}}$ was automatically corrected for the heat of dilution of $\mathrm{Ca}(\mathrm{ClO} 4)_{2}$ in $0.05 \mathrm{M} \mathrm{NaClO}_{4}$. After each experiment, the calorimeter was calibrated (simulated exothermic reaction) by passing an electric current $I$ through an electric calibration resistance with resistivity $R$ for a known time $t$. In a parallel series of experiments, the heat of dilution of the polymer from the initial concentration $C_{i}$ to the final concentration $C_{f}$ from experiments run at least in triplicate was determined and then subtracted from the experimental heat of mixing from the previous experiments. The final values of $\Delta \bar{H}^{\text {mixing }}\left(\mathrm{R}_{\mathrm{j}}\right)$ were normalized per mole of polymer repeating unit: the corresponded to heat exchanged upon the mixing a sodium pectate solution, at concentration $C_{f}$ in $0.05 \mathrm{M} \mathrm{NaClO}_{4}$, with $\mathrm{Ca}(\mathrm{ClO} 4)_{2}$ in aqueous $0.05 \mathrm{M} \mathrm{NaClO}_{4}$ at $\mathrm{R}_{\mathrm{j}}$ at $25{ }^{\circ} \mathrm{C}$. The calorimeter used gives an accuracy of the order of $2 \%$ for a rapid reaction having a heat absorption or evolution of $c a .2$ mcal. The limit in the accuracy and reproducibility of the data for such heat exchanges (as in the heat of mixing experiments), is mostly imposed by the calculation of the areas under the recorded peaks and by the slight drifts sometimes observed in the instrument baseline. The error in the dilution experiments with the polyelectrolytes in which heat exchanges of the order of less than 1 mcal had to be determined is of course larger. The uncertainty in the final enthalpy of dilution values here reported however never exceeded about $4 \%$. 
a)

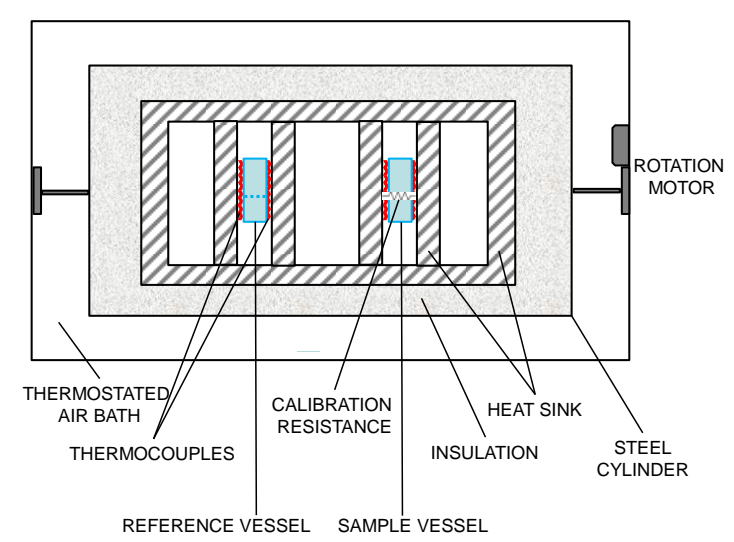

b)

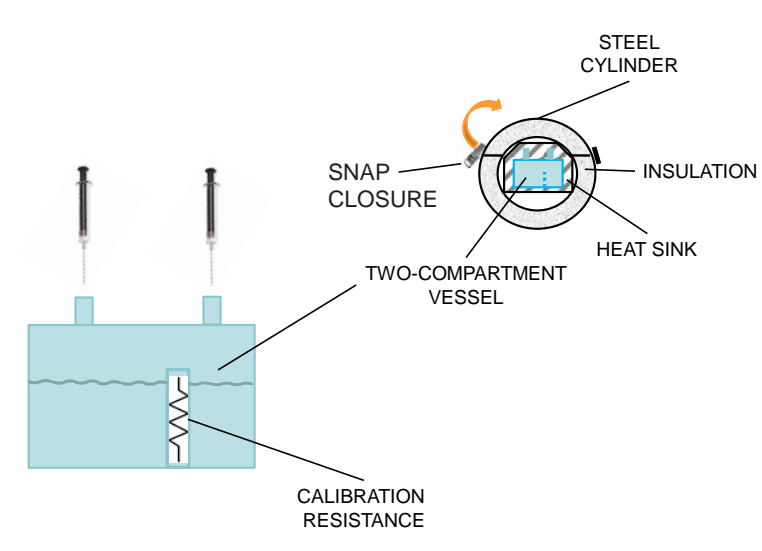

Scheme S1. Schematic representations of the batch microcalorimeter. a): plan (top) view of the calorimeter with fundamental parts. Sections of the cylinder containing the vessels (b) and of the sample vessel (c).

\section{f) dilatometric experiments}

The dilatometric experiments were performed using Linderstrøm-Lang dilatometers, whose application to study volume changes of biopolymer and polyelectrolyte solution was the pioneering work of Walter Kauzmann. It may be useful to report here the original description of the method by J.Rasper and W.Kauzmann" which was followed in our experiments: "Volume changes were measured by means of Carlsberg dilatometers similar to those described by Linderstrøm-Lang and Lanz $^{19}$ (see also Linderstrøm-Lang ${ }^{20}$ and Kauzmann ${ }^{21}$ ) with the modifications suggested by Johansen and Thygesen ${ }^{22}$ and by Johansen. ${ }^{23}$ The dilatometers are in the form of an inverted " $V$," with one arm containing the protein (usually about $5 \mathrm{ml}$. of a $5 \%$ solution) and the other containing the acid or base. All solutions are evacuated before pipetting into the dilatometers so as to reduce the amount of dissolved air, which otherwise often comes out of solution to form small bubbles when the dilatometers are placed in the thermostat. The space above the solutions is filled with purified kerosene and a graduated capillary $(10 \mu \mathrm{L}$ full scale, graduated to $0.05 \mu \mathrm{L}$. subdivisions; total length of graduations about $25 \mathrm{~cm}$.) is attached to the vertex of the " $V$ " by means of a specially lubricated ${ }^{23}$ ground glass joint. After adjusting the level of the kerosene in the capillary, the dilatometers are 
immersed in a well-controlled thermostat $\left( \pm 0.001^{\circ}\right)$, the level of the kerosene is read and the solutions are mixed by pouring back and forth from one arm of the " $V$ " to the other. The change in the kerosene level gives the volume change of the reaction. Volume changes are reproducible to a few hundredths of a microliter. All measurements were made at $30.00^{\circ} . "$ [References of the quotation from Reference 7 have not been reported here]. A schematic representation of the dilatometers is given in Scheme S2.

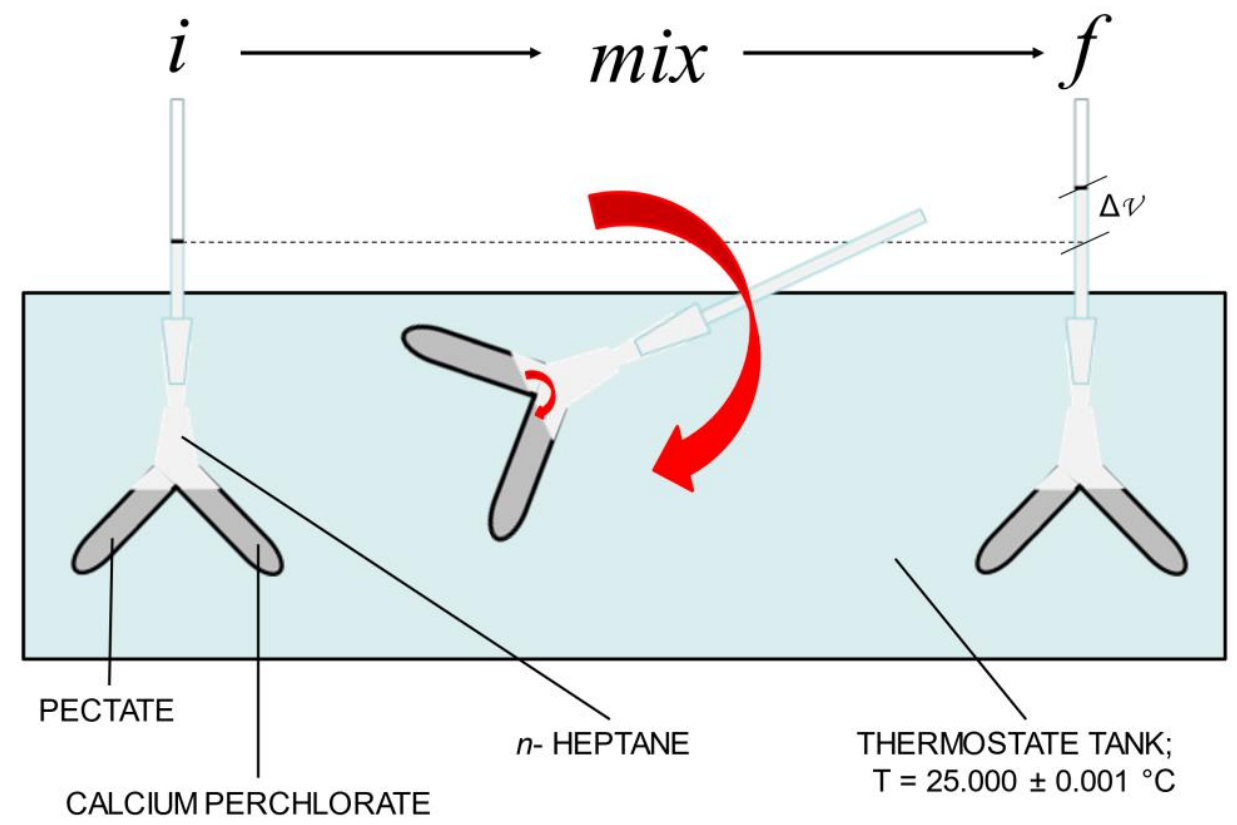

Scheme S2. Schematic representations of the setup for the dilatometric experiments. $i$ ) initial state until thermal equilibration after filling; $f$ ) final state until thermal equilibration after mixing.

The different details were: $i$ ) the pectate solution in aqueous $\mathrm{NaClO}_{4} 0.05 \mathrm{M}$ which was in one arm was much more dilute (typically about $0.15 \%$ ) than the protein solution $(5 \%)$; $i$ ) the other arm contained a solution of $\mathrm{Ca}\left(\mathrm{ClO}_{4}\right)_{2}$ in $\mathrm{NaClO}_{4} 0.05 \mathrm{M}$ at the concentration such as to achieve the desired $\mathrm{R}_{\mathrm{j}}$ value; iii) $n$-heptane (Carlo Erba Reagents - Pro Analysis) was used instead of kerosene: it was purified sequentially according to the procedure of Katz \& Ferris ${ }^{8}$ with concentrated sulfuric acid, $10 \% \mathrm{KMnO}_{4}$ in $10 \% \mathrm{KOH}, 10 \% \mathrm{KMnO}_{4}$ in $10 \%$ sulfuric acid, and distilled water, and redistilled. This was equilibrated against water prior to use; iiii) the temperature was $25^{\circ} \mathrm{C}$. In two series of parallel experiments the volume changes of dilution $v s$. $\mathrm{NaClO}_{4} 0.05 \mathrm{M}$ of solutions of pectate or $\mathrm{Ca}\left(\mathrm{ClO}_{4}\right)_{2}$ - both in $\mathrm{NaClO}_{4} 0.05 \mathrm{M}$ and at the same concentration of the mixing experiments - were performed. Those measured values (which were usually much smaller than the experimental volume changes of mixing pectate and calcium) were eventually subtracted from the latter ones. 


\section{g) circular dichroism (CD) experiments}

Circular dichroism (CD) measurements were performed with a Jasco J-500A dichrograph, equipped with a DP-500 data processor using thermostated quartz cells. At least four spectra were cumulated in the 200-250 nm region after optimization of the cell pathlength, transmittance and CD signal parameters.

\section{h) fitting of the circular dichroism (CD) data from Reference 3.}

The chiro-optical data used for the evaluation of the fraction of bonded $\mathrm{Ca}^{2+}$ ions, $\theta$, have been taken from reference ${ }^{3}$. Addition of calcium ions to a polyuronate solution (at a concentration, $C_{p}$, equal to $0.0035 \mathrm{~mol}$ repeating unit $\cdot \mathrm{L}^{-1}$ ) brings about a significant change of the molar ellipticity in the UV region. ${ }^{1,9}$ To highlight the quantitative effect of the chiro-optical perturbation induced by calcium and to get rid of the sign of the molar ellipticity of calciumfree pectate solution, and of the calcium-induced variation, it was resorted to take the absolute value of the relative change of molar ellipticity, $S[\vartheta]$, at $\lambda=210 \mathrm{~nm}$, which is a function of the molar ratio of total calcium added to polymer repeating units, $\mathrm{R}_{\mathrm{j}}$ :

$$
S[\vartheta]\left(R_{j}\right)=\left|\frac{[\vartheta]\left(R_{j}\right)-[\theta]_{R_{j}=0}}{[\vartheta]_{R_{j}=0}}\right|=1-\left|\frac{[\vartheta]\left(R_{j}\right)}{[\vartheta]_{R_{j}=0}}\right|
$$

Moreover,

$$
S[\vartheta]\left(R_{j}\right)=\Delta B_{[\vartheta]} \cdot \theta\left(R_{j}\right)
$$

where $\Delta B_{[\vartheta]}$ is the (absolute) fractional change of molar ellipticity of the elementary process, a dimensionless intensive property, specific of each polyuronate, and $\theta\left(R_{j}\right)$ is the value of the fraction of bonded $\mathrm{Ca}^{2+}$ ions, $\theta$, at $\mathrm{R}_{\mathrm{j}}$.

Given the clearly monomodal dependence of $S[\vartheta]\left(R_{j}\right)$ on $\mathrm{R}_{\mathrm{j}}$, to evaluate $\theta\left(R_{j}\right)$ it was resorted to make use of the Langmuir equation, which for the specific case would read:

$$
\begin{aligned}
S[\vartheta]\left(R_{j}\right) & =\Delta B_{[\theta]} \cdot \frac{\left[\mathrm{Ca}^{2+}\right] \cdot K_{\text {ass }}}{1+\left[\mathrm{Ca}^{2+}\right] \cdot K_{a s s}}=\Delta B_{[\vartheta]} \cdot \frac{R_{j} \cdot C_{p} \cdot K_{\text {ass }}}{1+R_{j} \cdot C_{p} \cdot K_{a s s}}= \\
& =\Delta B_{[\vartheta]} \cdot \frac{R_{j}}{1 /\left(C_{p} \cdot K_{a s s}\right)^{+R_{j}}}
\end{aligned}
$$

$\left[\mathrm{Ca}^{2+}\right]$ is the total molar concentration of added calcium ions, and $K_{a s s}$ is the association constant of the adsorbate (i.e. the calcium ion) for the surface (i.e. the polymer) according to 
the Langmuir adsorption model. Fitting of the data points of Figure 1 using equation $<\mathrm{S} 4>$, returned for the two fitting parameters the values $\Delta B_{[\vartheta]}=0.98$ and $K_{a s s}=1232\left(\mathrm{R}^{2}=0.99553\right)$. As already pointed out in reference ${ }^{3}$, the fact that $\Delta B_{[\vartheta]}=0.98$, i.e. very close to 1 , is purely incidental, inasmuch as it just reflects the specific value of the (absolute) fractional change of molar ellipticity of pectate upon addition of calcium at $\lambda=210 \mathrm{~nm}$.

\section{Section 2. More on the thermodynamics of binding.}

a) the reference state of the specific affinity thermodynamic functions

In equations $<4>$ to $<7>$ of the main paper, the terms $F\left(\mathrm{R}_{\mathrm{j}}\right)\left(F\left(\mathrm{R}_{\mathrm{j}}\right)=\left(r_{\text {cond }}\right)^{\text {tot }}\left(\mathrm{R}_{\mathrm{j}}\right) \cdot \Delta \overline{\mathrm{X}}^{\text {aff }}\right.$, where $X=G, H, S, V$ ) account for the specific affinity contributions to the total energy of the system assuming $\mathrm{Na}^{+}$-pectate as the "reference" state. Strictly speaking, the correct reference counterion is a dimensionless one, devoid of any hydration, showing only electrostatic interactions with an equally theoretical mono-dimensional array of negative charges, i.e. the polyanion. Any real counterion will depart from this scheme.

The explicit expression for the $F\left(\mathrm{R}_{\mathrm{j}}\right)$ is (recalling that symbol $i$ refers to monovalent counterions, and symbol $j$ refers to divalent counterions):

$$
\begin{gathered}
F\left(\mathrm{R}_{\mathrm{j}}\right)=F^{i}\left(\mathrm{R}_{\mathrm{j}}\right)+F^{j}\left(\mathrm{R}_{\mathrm{j}}\right) \\
F^{i}\left(\mathrm{R}_{\mathrm{j}}\right)=\left(\left(r_{\text {cond }}^{i}\left(\mathrm{R}_{\mathrm{j}}\right)-r_{\text {cond }}^{i}\left(\mathrm{R}_{\mathrm{j}}=0\right)\right) \cdot\left(\overline{\mathrm{X}}^{\text {free }, i}-\overline{\mathrm{X}}^{\text {cond }, i}\right)\right)= \\
=\Delta r_{\text {cond }}^{i} \cdot\left(-\Delta \overline{\mathrm{X}}^{\text {aff }, i}\right)
\end{gathered}
$$

and

$$
\begin{aligned}
F^{j}\left(\mathrm{R}_{\mathrm{j}}\right) & =\left(\left(r_{\text {cond }}^{j}\left(\mathrm{R}_{\mathrm{j}}\right)-r_{\text {cond }}^{j}\left(\mathrm{R}_{\mathrm{j}}=0\right)\right) \cdot\left(\overline{\mathrm{X}}^{\text {free }, j}-\overline{\mathrm{X}}^{\text {cond }, j}\right)\right)= \\
& =\Delta r_{\text {cond }}^{j} \cdot\left(\Delta \overline{\mathrm{X}}^{\text {aff }, j}\right)
\end{aligned}
$$

The negative sign in front of $\Delta \overline{\mathrm{X}}^{a f f, i}$ in equation $\langle\mathrm{S} 6\rangle$ stems from the fact the monovalent counterions are released form the condensation volume upon binding, losing any specific affinity effect. If the monovalent counterions is assumed as the "reference" counterion, then $-\Delta \overline{\mathrm{X}}^{a f f, i} \equiv 0, F^{i}\left(\mathrm{R}_{\mathrm{j}}\right)=0$ and equation $\langle\mathrm{S} 5>$ reduces to:

$$
F\left(\mathrm{R}_{\mathrm{j}}\right)=F^{j}\left(\mathrm{R}_{\mathrm{j}}\right)=\Delta r_{\text {cond }}^{j} \cdot\left(\Delta \overline{\mathrm{X}}^{a f f, j}\right)=\left(r_{\text {cond }}\right)^{t o t}\left(\mathrm{R}_{\mathrm{j}}\right) \cdot \Delta \overline{\mathrm{X}}^{\text {aff }}
$$


that is the formalism of equations $\langle 4\rangle$ to $\langle 7\rangle$, having dropped the index $j$ since only divalent counterions are then supposed to show specific affinity interactions with the (real) polyelectrolyte.

\section{b) a model of the polymer and complex contributions to calcium bonding}

The analysis presented in the paper has consistently led to identify the intensive values of four different thermodynamic parameters, namely $\Delta \bar{G}_{1}, \Delta \bar{G}_{2}, \Delta \bar{H}_{1}, \Delta \bar{H}_{2}$, (and the corresponding values of $\Delta \bar{S}_{1}$ and $\Delta \bar{S}_{2}$ ), together with the $\theta$-dependence of $\Delta \bar{S}_{\text {network }}(\theta)$; they correspond to the initial, or type-1, mode of strong calcium binding, "1", to the type-2 or "cluster" mode of bonding, " 2 ", and to the extensive network formation following interchain association caused by the increasing amount of "linkers", respectively.

Little doubt can be cast upon the idea that the thermodynamic parameters of the two former types of calcium bonding are the result of different short-range interactions that contribute to the observed behavior. Having already said of the purely entropic origin of the long-range interactions $\left(\Delta \bar{S}_{\text {network }}(\theta)\right)$, attention is hereafter given to those short-range type of interactions, namely to processes " 1 " and " 2 ". A question arises from inspection of the corresponding thermodynamic parameters in Table 1 of the paper: "What makes type- 2 mode of binding (which becomes the dominant one to stabilize sequences of calcium linkers in between facing pectate chains upon increasing the amount of added calcium) a thermodynamically less favored process compared with type-1 mode?". The goal of this Section is to try to give possible hints to help answering that question.

To further analyze the short-range interactions of bonding modes "1" and "2", one can schematically separate terms which essentially pertains to the formation of the complex, made of uronate ligand(s) around a coordinated calcium ion ("complex"), and those which more clearly correlate with the chelating polyuronate chain(s) ("polymer").

The former ones, in turn, can be schematically attributed $i$ ) to effects connected with the desolvation of both the (calcium) ion and the carbohydrate coordinating groups (e.g. the carboxylate or the hydroxyl groups of the polyuronate chain) and $i$ ) to the formation of specific coordination bonds in the "inner sphere", highly desolvated, complex, to such an extent as to locally producing charge annihilation. Nevertheless, in the following, it will not be tried to separate the two contributions to complex formation.

The interactions hereafter referred to as "polymer" certainly include conformational changes of the main chain glycosidic linkages and either intramolecular or intermolecular hydrogen-bond formation, depending on the molecularity of the process. 
In general, it will hold:

$$
\Delta \bar{G}_{1}=\Delta \bar{G}_{1}^{\text {complex }}+\Delta \bar{G}_{1}^{\text {polymer }}
$$

and

$$
\Delta \bar{G}_{2}=\Delta \bar{G}_{2}^{\text {complex }}+\Delta \bar{G}_{2}^{\text {polymer }}
$$

Similar equation will be supposed to hold for $\Delta \bar{H}$ and $\Delta \bar{S}$, not reported to avoid triviality. At this point a somewhat speculative - albeit not a-priori rejectable - hypothesis can be made, namely, to borrow from known cases of conformational transition/association processes of ionic polysaccharides the thermodynamic values to attribute to the "polymer" component of bonding. It should be looked upon as a "simulation exercise" whose only merit may be that of stimulating further work to deepen this aspect. Starting from type-1 bonding, one should recall both $i$ ) that the orientation of the two chains across the point-like cross-link is supposed to be not far from $90^{\circ}$ ("tilted"), as prescribed by both the Borukhov's and the "tilted egg-box" models, on one side, and ii) that $\Delta B_{[\vartheta]}$ in type-1 bonding has already attained its maximum value. It looks almost mandatory then to assume that each of the two crossing disaccharides chelating the calcium ion has undergone the "loose-2 1 -helix"-to-"tight-21-helix" intramolecular transition as discussed in the Conclusion part of the paper. The first step of the simulation then pertains to searching for a similar "loose" to "tight" intramolecular transition and to attribute its thermodynamic values to the "polymer" component of type-1 bonding. The thermodynamic data of two cases (both pertaining to the transition for a "loose-helix" - disorder - to a final $3_{1}$ helix - order) are at hand, namely those of the $\mathrm{H}^{+}$-induced conformational transition of sodium pectate $^{2}$ and that of the intramolecular transition of $\kappa$-carrageenan $(d i s \rightarrow 1 h) .{ }^{10}$ The corresponding parameters are: $\Delta \bar{H}_{\text {pectate }}^{\text {conf.trans. }}=-70_{0}$ cal $\cdot$ mole galacturonate ${ }^{-1}$ and $\Delta \bar{S}_{\text {pectate }}^{\text {conf.trans. }}=-2.2 \mathrm{cal} \cdot$ mole galacturonate ${ }^{-1} \mathrm{~K}^{-1}$ for $\mathrm{Na}^{+}$-pectate, and $\Delta \bar{H}_{\text {carrag. } 1 \mathrm{~h}}^{\text {conf.trans }}=-1348 \mathrm{cal}$ - mole carrabiose ${ }^{-1}$ and $\Delta \bar{S}_{\text {carrag. }}^{\text {conf } 1 \mathrm{~h}}$. $=-3.8 \mathrm{cal} \cdot$ mole carrabiose $^{-1} \mathrm{~K}^{-1}$ for $\kappa$-carrageenan, respectively. When normalized per four galacturonate units (as required by the stoichiometry of the calcium bonding ${ }^{3}$ ) those values are: $\Delta \bar{H}_{\text {pectate }}^{\text {conf.trans. }}=-28_{00}$ cal $\cdot$ mole complex $^{-1}$ and $\Delta \bar{S}_{\text {pectate }}^{\text {conf.trans. }}=-8.8 \mathrm{cal} \cdot$ mole complex ${ }^{-1} \mathrm{~K}^{-1}$ for $\mathrm{Na}^{+}$-pectate, and $\Delta \bar{H}_{\text {carrag. } 1}^{\text {conf. }}=-2696 \mathrm{cal} \cdot \mathrm{mole}$ complex $^{-1}$ and $\Delta \bar{S}_{\text {carrag. } 1 \mathrm{~h}}^{\text {conf.trans. }}=-7.6 \mathrm{cal} \cdot$ mole complex ${ }^{-1} \mathrm{~K}^{-1}$ for $\kappa$-carrageenan, respectively. The values of the thermodynamic functions of two - apparently very different - ionic galactans are surprisingly similar. This observation has prompted to assume their average values to be used 
as $\Delta \bar{H}_{1}^{\text {polymer }}$ and $\Delta \bar{S}_{1}^{\text {polymer }}$, respectively. They are reported in Table $\mathrm{S} 2$, together with the corresponding value of $\Delta \bar{G}_{1}^{\text {polymer }}$. (Incidentally, those values are in reasonable agreement also with those determined across the temperature-induced transition of the protonated form - albeit partly associated - to the disordered one of pectate using DSC data: ${ }^{5} 33_{60} \mathrm{cal} \cdot(\mathrm{mol} \text { complex})^{-}$ ${ }^{1}$ and $11.6 \mathrm{cal} \cdot(\mathrm{mol} \text { complex })^{-1} \cdot \mathrm{K}^{-1}$, respectively, once properly re-normalized; moreover, the $\Delta \bar{H}^{\text {trans. }}$ (disorder-to-order) of the uncharged galactan agarose - after proper normalization is $\left.\Delta \bar{H}^{\text {trans. }}=-26_{70} \mathrm{cal} \cdot(\mathrm{mol} \text { complex })^{-1} \cdot{ }^{11}\right)$ The value of the thermodynamic functions pertaining to the "complex" formation have been calculated in a straightforward way by subtracting the "polymer" value from the integral values pertaining to the type-1 process. Also those values have been reported in Table $\mathrm{S} 2$.

The situation regarding type- 2 mode of bonding is less straightforward. The only case very similar to the (antiparallel) pairing of "egg-box" sequences of calcium-pectate is that of the side-by-side pairing $(1 \mathrm{~h} .1 \mathrm{~h})$ of stretches of (antiparallel) single helices of $\kappa$-carrageenan interacting with $\mathrm{K}^{+}$or $\mathrm{Cs}^{+}$counterions. ${ }^{10}$ The thermodynamic parameters are: $\Delta \bar{H}_{\text {carrag. } 1 \mathrm{c} .1 \mathrm{~h}}^{\text {conf }}=$ - $44_{65} \mathrm{cal} \cdot$ mole carrabiose ${ }^{-1}$ and $\Delta \bar{S}_{\text {carrag. } 1 h .1 h}^{\text {conf.trans. }}=-11.4 \mathrm{cal} \cdot$ mole carrabiose ${ }^{-1} \mathrm{~K}^{-1}$, which, upon re-normalization for the $\mathrm{Ca}^{2+}$-bonding, give: $\Delta \bar{H}_{2}^{\text {polymer }}=-89_{30} \mathrm{cal} \cdot$ mole complex ${ }^{-1}$ and $\Delta \bar{S}_{2}^{\text {polymer }}=-22.8 \mathrm{cal} \cdot$ mole complex ${ }^{-1} \mathrm{~K}^{-1}$, respectively; also they are reported in Table $\mathrm{S} 2$, together with the calculated values of $\Delta \bar{H}_{2}^{\text {complex }}$ and $\Delta \bar{S}_{2}^{\text {complex }}$, and the ensuing values of $\Delta G_{2}^{\text {polymer }}$ and $\Delta G_{2}^{\text {complex }}$.

Inspection of the Gibbs free energy values returns the macroscopic evidence that, albeit both being always favorable, the relative contributions of the "polymer" and the "complex" processes are extremely different: whereas the "complex" component accounts for $92 \%$ of $\Delta \bar{G}_{1}$ the situation completely reverts for $\Delta \bar{G}_{2}$ where the "polymer" contribution accounts for $93 \%$. In other words, the simulation suggests that the driving force of the " 1 " mode of bonding is almost completely due to the stability of the point-like complex with calcium; the marginal contribution of the "polymer" component looks like an unescapable consequence of the "conformational change propensity" of the pectate backbone, as addressed in the Conclusion section of the paper. The sign of both the enthalpic and the entropic parts of the "complex" component are always positive, both for the type- 1 and the type- 2 processes. However, in "1" the value of $T_{m}\left(T_{m}=\Delta \bar{H} / \Delta \bar{S}\right)$ is $70 \mathrm{~K}$, very close to (actually, even smaller than) the value of $T_{m}$ for the affinity process, which is similarly characterized by positive values of $\Delta \bar{H}$ and $\Delta \bar{S}$ as well as $\Delta \bar{V}$ - due to extensive desolvation. The use of $T_{m}$ as a qualitative marker of the nature 
of a common molecular process is rooted in the so-called Barclay-Butler type of relationship, sometimes ambiguously indicated as "entropy-enthalpy compensation". ${ }^{12}$ The value of $\Delta \bar{V}$ in the bonding processes is even larger, producing larger (positive) desolvation contributions to the stability of the complex; nevertheless, negative contributions deriving from "coordination" of calcium (i.e. the very likely formation of new ligand-ion bonds) must be invoked to explain the otherwise inexplicable lower values of $\Delta H_{1}^{\text {complex }}$ and $\Delta S_{1}^{\text {complex }}$ with respect to the affinity process.

The postulated modeling of the components to bonding tells that interchain "alignment-andbonding" is at the root of the stability of mode " 2 "; over and above an intramolecular conformational transition (as taught by CD) a massive network of intermolecular H-bonds develops among the two parallel polymer stretches. In the proposed scheme, it brings about an extra (negative) contribution to $\Delta \bar{H}^{\text {polymer }}$ of more than $6 \mathrm{kcal} \cdot\left(\right.$ mole complex) ${ }^{-1}$; albeit paralleled by a loss of entropy of almost $15 \mathrm{cal} \cdot(\text { mole complex })^{-1} \mathrm{~K}^{-1}$, it still produces a net gain of $\Delta \bar{G}$. Very interestingly, there is a difference of only $1.1 \%$ between $\left(\Delta \bar{H}_{2}-\Delta \bar{H}_{1}\right)$ and $\left(\Delta H_{2}^{\text {polymer }}-\Delta H_{1}^{\text {polymer }}\right.$ - see box ("2"-“1”) in Table S2), suggesting that the choice of the "polymer" component in step " 2 " is likely to be very sound. Also in this case the values of the thermodynamic parameters of the "complex" process are very informative: whereas the value of $\Delta H_{2}^{\text {complex }}$ is only marginally different from $\Delta H_{1}^{\text {complex }}$ (about $+7 \%$ ), the value of $\Delta S_{2}^{\text {complex }}$ suffers a loss of about $71 \%$ with respect to $\Delta S_{1}^{\text {complex }}$. From the standpoint of the complex formation, one might possibly envisage a situation in which in " 1 " a significant flexibility of the ligand(s) favors the optimal mutual steric arrangement of the ion and its ligands in the complex. At variance, the great stability of the "shifted egg-box" in "2" produces a very rigid "cage" for the coordinated calcium ion, which pays the cost of being a "caged ion" by loosing much of its motional freedom as an important loss of entropy.

As openly admitted at the beginning of this Section, all the above discussion is just a simulation of the possible components to the bonding steps of calcium by sodium poly(galacturonate). It should be looked upon as a mere stimulus to further studies, ideally using different techniques, to enlarge the molecular understanding of such an important counterion-polyion system. 
Table S2

\begin{tabular}{|c|c|c|c|c|c|c|c|c|c|}
\hline Thermodyn. prop. & $\Delta \bar{G}^{0}$ & $\Delta \bar{H}^{0}$ & $-T \cdot \Delta \bar{S}^{0}$ & $\Delta \bar{S}^{0}$ & $\Delta \bar{V}^{0}$ & $n_{W}{ }^{*}$ & $\mathrm{~T}_{\mathrm{m}}$ & $K$ & $\log (K)$ \\
\hline interaction & \multicolumn{3}{|c|}{$\mathrm{cal} \cdot(\text { mole complex })^{-1}$} & $\begin{array}{c}\text { cal } \cdot(\text { mole } \\
\text { complex })^{-1} \\
\mathrm{~K}^{-1}\end{array}$ & $\begin{array}{c}\mathrm{cm}^{3} \cdot \\
(\text { mole } \\
\text { complex })^{-1}\end{array}$ & $\begin{array}{c}\text { moles of } \\
\text { released } \\
\text { water }(\text { mole } \\
\text { complex })^{-1}\end{array}$ & $\mathrm{~K}$ & $e^{-\frac{\Delta G^{\circ}}{R \cdot T}}$ & \\
\hline affinity & $-41_{35}$ & $15_{00}$ & $-56_{35}$ & 18.9 & 12 & 6.7 & 79 & 1074 & 3.03 \\
\hline Type-1, "1" & -3744 & $-17_{04}$ & -2040 & 6.8 & 29 & 16.1 & n.a. & 555 & 2.74 \\
\hline polymer & -310 & $-27_{48}$ & 2438 & -8.2 & 0 & 0 & 336 & 1.7 & 0.23 \\
\hline complex & -3434 & $10_{44}$ & $-44_{78}$ & 15.0 & 29 & 16.1 & 70 & 329 & 2.52 \\
\hline Type-2, “2”' & -2276 & $-78_{17}$ & $55_{41}$ & -18.6 & 29 & 16.1 & 421 & 47 & 1.67 \\
\hline polymer & $-21_{18}$ & $-89_{30}$ & $68_{12}$ & -22.8 & 0 & 0 & 391 & 36 & 1.55 \\
\hline complex & $-1_{58}$ & $11_{13}$ & $-12_{71}$ & 4.2 & 29 & 16.1 & 262 & 1.3 & 0.12 \\
\hline "2" - "1" & $14_{69}$ & $-61_{13}$ & $75_{81}$ & -25.4 & 0 & 0 & 240 & 0.084 & -1.08 \\
\hline polymer & $-18_{08}$ & $-61_{82}$ & $43_{75}$ & -14.7 & 0 & 0 & 421 & 21.1 & 1.33 \\
\hline complex & $32_{76}$ & 69 & $32_{06}$ & -10.7 & 0 & 0 & n.a. & 0.00397 & -2.40 \\
\hline
\end{tabular}

Table S2. Thermodynamic parameters of the fundamental short-range processes accompanying calcium bonding (i.e. type1 and type-2) and of the corresponding polymer and complex components.

\section{References}

(1) Paoletti, S.; Cesaro, A.; Delben, F.; Ciana, A. Ionic Effects on the Conformation, Equilibrium, Properties, and Rheology of Pectate in Aqueous Solutions and Gels. In Chemistry and Function of Pectins; ACS Symposium Series; American Chemical Society, 1986; Vol. 310, pp $7-73$.

(2) Cesàro, A.; Ciana, A.; Delben, F.; Manzini, G.; Paoletti, S. Physicochemical Properties of Pectic Acid. I. Thermodynamic Evidence of a pH-Induced Conformational Transition in Aqueous Solution. Biopolymers 1982, 21 (2), 431-449.

(3) Donati, I.; Benegas, J. C.; Paoletti, S. Polyelectrolyte Study of the Calcium-Induced Chain Association of Pectate. Biomacromolecules 2006, 7 (12), 3439-3447.

(4) Bongaerts, K.; Paoletti, S.; Denef, B.; Vanneste, K.; Cuppo, F.; Reynaers, H. Light Scattering Investigation of -Carrageenan Aqueous Solutions. Concentration Dependence of Association. Macromolecules 2000, 33 (23), 8709-8719.

(5) Gilsenan, P. M.; Richardson, R. K.; Morris, E. R. Thermally Reversible Acid-Induced Gelation of Low-Methoxy Pectin. Carbohydr. Polym. 2000, 41 (4), 339-349.

(6) Gamini, A.; Civitarese, G.; Cesàro, A.; Delben, F.; Paoletti, S. Gelation Mechanism of Ionic 
Polysaccharides. Makromol. Chemie. Macromol. Symp. 1990, 39 (1), 143-154.

(7) Rasper J. and Kauzmann W. Volume Changes in Protein Reactions. I. Ionization Reactions of Proteins. J.Am.Chem.Soc. 1962, 84 (10), 1771 - 1777.

(8) Katz, S.; Ferris, T. G. Dilatometric Study of the Interactions of Bovine Serum Albumin with Urea*. Biochemistry 1966, 5 (10), 3246-3253.

(9) Donati, I.; Gamini, A.; Skjåk-Bræk, G.; Vetere, A.; Campa, C.; Coslovi, A.; Paoletti, S. Determination of the Diadic Composition of Alginate by Means of Circular Dichroism: A Fast and Accurate Improved Method. Carbohydr. Res. 2003, 338 (10).

(10) Paoletti, S.; Gamini, A.; Vetere, A.; Benegas, J. C. Metal Ion - Ionic Polysaccharide Interactions: Theoretical Developments and Experimental Results. Macromol. Symp. 2002, $186(1), 141-146$.

(11) Rochas, C. Calorimetric Study of Galactans. Food Hydrocoll. 1987, 1 (3), 215-225.

(12) Barclay, I. M.; Butler, J. A. V. The Entropy of Solution. Trans. Faraday Soc. 1938, 34 (0), $1445-1454$. 